\title{
Prediction of mortality in severe dengue cases
}

\author{
Saiful Safuan Md-Sani ${ }^{1}$, Julina Md-Noor ${ }^{2 *}$, Winn-Hui Han', Syang-Pyang Gan ${ }^{1}$, Nor-Salina Rani ${ }^{1}$, Hui-Loo Tan ${ }^{1}$, \\ Kanimoli Rathakrishnan ${ }^{1}$, Mohd Azizuddin A-Shariffuddin ${ }^{3}$ and Marzilawati Abd-Rahman ${ }^{1}$
}

\begin{abstract}
Background: Increasing incidence of dengue cases in Malaysia over the last few years has been paralleled by increased deaths. Mortality prediction models will therefore be useful in clinical management. The aim of this study is to identify factors at diagnosis of severe dengue that predicts mortality and assess predictive models based on these identified factors.

Method: This is a retrospective cohort study of confirmed severe dengue patients that were admitted in 2014 to Hospital Kuala Lumpur. Data on baseline characteristics, clinical parameters, and laboratory findings at diagnosis of severe dengue were collected. The outcome of interest is death among patients diagnosed with severe dengue.

Results: There were 199 patients with severe dengue included in the study. Multivariate analysis found lethargy, OR 3.84 (95\% Cl 1.23-12.03); bleeding, OR 8.88 (95\% Cl 2.91-27.15); pulse rate, OR 1.04 (95\% Cl 1.01-1.07); serum bicarbonate, OR 0.79 (95\% Cl 0.70-0.89) and serum lactate OR 1.27 (95\% Cl 1.09-1.47), to be statistically significant predictors of death. The regression equation to our model with the highest AUROC, 83.5 (95\% Cl 72.4-94.6), is: Log odds of death amongst severe dengue cases $=-1.021-0.220$ (Serum bicarbonate) $+0.001($ ALT) +0.067 (Age) 0.190(Gender).

Conclusion: This study showed that a large proportion of severe dengue occurred early, whilst patients were still febrile. The best prediction model to predict death at recognition of severe dengue is a model that incorporates serum bicarbonate and ALT levels.
\end{abstract}

Keywords: Severe dengue, Mortality, Predict

\section{Background}

Dengue infection has occurred in Malaysia for over a century [1]. Since the 1980s, outbreaks have been increasing in frequency [2]. Dengue is now accepted as endemic in Malaysia for the last 20 years. Despite massive efforts by various central government departments managing public health and many other agencies of the local government to curb it, the number of dengue cases reported continues to increase, and a rise in mortality has paralleled that increase. A national key performance indicator was established; a case fatality rate of less than $0.2 \%$ was targeted.

\footnotetext{
* Correspondence: julinamn@gmail.com

${ }^{2}$ Faculty of Medicine, Universiti Teknologi MARA (UiTM), Jalan Hospital, 47000

Sungai Buloh, Selangor, Malaysia

Full list of author information is available at the end of the article
}

An epidemiological analysis in Malaysia revealed that all four DENV serotypes were found to be co-circulating during the period 2000-2012, although the predominant serotypes varied over time, both nationally and within the individual states in Malaysia [3]. An example of this variation occurred in the year 2014 when the predominant serotype had switched twice from DENV-2 to DENV-1, in February and June [4]. Prior to 2013, the case fatality rates were less than $0.2 \%$, which was within the national target. However, from 2013 onwards, dengue incidence and case fatality rate started to increase and in 2015 had almost doubled [5].

Along with this increase in burden of disease the management of dengue, especially severe dengue, continues to baffle physicians. Despite continuous efforts to upgrade and improve clinical practice guidelines, physicians are confronted with patients who do not fall neatly

(C) The Author(s). 2018 Open Access This article is distributed under the terms of the Creative Commons Attribution 4.0 International License (http://creativecommons.org/licenses/by/4.0/), which permits unrestricted use, distribution, and 
into typical description of the disease, hence pose a clinical conundrum. The spectrum of dengue infection ranges from asymptomatic to mild febrile illness, dengue with warning signs and severe dengue. Severe dengue is defined as end organ involvement, severe bleeding and/ or severe plasma leakage. Although only a small percentage of patients went on to develop severe dengue, with a study quoting a proportion of $5 \%$ of all dengue infections studied [6], these severe dengue carries significant mortality. Mortality in dengue is believed to be potentially preventable. Dengue is a dynamic, systemic illness and physicians have always found themselves summing up best estimates based on personal experience.

In that respect, additional knowledge would be indispensable in the management of dengue patients. Many studies have attempted to predict those that will develop severe dengue using clinical features and laboratory findings. There have also been studies that examined inflammatory markers, cytokines and genetic markers in predicting severity of dengue [7]. However, we believe that there is a need to refine this further - to predict mortality among those diagnosed to have severe dengue. We therefore aimed to identify factors at diagnosis of severe dengue that predict mortality and, built and assess predictive models based on these identified factors.

\section{Methods}

The study was approved by the Medical Research and Ethics Committee (MREC), Ministry of Health of Malaysia (Research ID NMRR-15-2023-24,849). As the study involved data collection from case notes only, the MREC granted a waiver of informed consent. Our report is based on the STrengthening the Reporting of OBservational studies in Epidemiology (STROBE) 2015 guideline.

The study was a retrospective study of adult patients with severe dengue who were admitted into Hospital Kuala Lumpur throughout 2014. Sample recruitment was conducted from 1 January 2014 until 31 December 2014. Data were extracted from case notes of selected patients who fulfilled the study's inclusion criteria. A data collection pro forma was used to ensure integrity of data. As the information in the case notes were information used in patients' management, and thus would have been corrected for errors by managing clinicians, therefore we considered the information in the case notes as accurate.

Patients were selected for inclusion if, 1) they were $\geq$ 18 years old, 2) their presentation satisfied our criteria for suspected dengue, 3 ) the case fulfilled our definition of severe dengue, and 4) the presence of dengue viral infection was confirmed via NS1 antigen, high-titre level of IgG or positive IgM, from an admission serum sample. Our criteria for suspected dengue, based on the World Health Organization (WHO) 2009 criteria, were fever plus any two of: 1) aches and pain, 2) nausea and/ or vomiting, 3) rash, 4) leucopenia, or 5) presence of any warning signs. The definition of severe dengue in our study was also based on WHO 2009 definition. We defined severe dengue by presence of any one of the following: 1) decompensated shock due to severe plasma leakage, 2) compensated shock due to severe plasma leakage, 3) respiratory compromise due to severe plasma leakage, 4) severe hepatitis, 5) severe bleeding that required intervention, or 6) severe organ involvement such as acute kidney injury defined by elevated serum creatinine (according to gender-specific levels), myocarditis or encephalopathy. Decompensated shock was defined by presence of systolic blood pressure (SBP) less than $90 \mathrm{mmHg}$, or mean arterial pressure (MAP) of less than $65 \mathrm{mmHg}$, or a drop in systolic blood pressure of more than $40 \mathrm{mmHg}$ from patient's known usual baseline readings. Compensated shock required signs of impaired peripheral perfusion, occurring in combination rather than singly, in presence of systolic blood pressure of $\geq 90 \mathrm{mmHg}$. Severe hepatitis was defined as AST level $\geq$ $1000 \mathrm{IU} / \mathrm{L}$, or ALT level $\geq 1000 \mathrm{IU} / \mathrm{L}$.

Clinical management at Hospital Kuala Lumpur followed the Malaysian Clinical Practice Guideline on Management of Dengue Infection in Adults 2010 and the WHO 2009 clinical practice guideline for dengue.

Our outcome of interest was mortality among patients diagnosed with severe dengue. Patients were grouped into those who died and those who survived. We reviewed case notes to collect data. Data collected were baseline characteristics, clinical parameters, laboratory findings and time of events. Times of events were: dates and time of fever onset, time of admission, time of diagnosis of severe dengue, time of defervescence (start of temperature persistently $<38{ }^{\circ} \mathrm{C}$ ) and time of occurrence of outcome. Clinical and laboratory parameters that were closest to the time of diagnosis of severe dengue were collected. These variables formed as candidate predictors for our prediction model. We also collected data on nadir and highest levels of relevant laboratory parameters, along with their timings, for descriptive purposes. Data on types of severe dengue diagnoses that occurred in a patient were also collected.

We calculated that a sample size of 13 deaths was needed for this study for an AUROC of 70\%, at confidence level of $95 \%$ and power of $80 \%$. Analyses were performed in SAS University Edition (Copyright ( $)$ 2012-2016, SAS Institute Inc., Cary, NC, USA). Continuous variables were tested for uniformity using the KolmogorovSmirnov test and normality with the Shapiro-Wilk test. As our data were mostly non-parametric, we used non-parametric analyses for data interrogation. Categorical variables were expressed as frequencies and percentages. Continuous variables with non-normal 
distribution were summarised as median and interquartile range (IQR).

Identification of all factors that were significantly associated with mortality was made using the Chi-square test or Fisher's exact test for categorical variables and Mann-Whitney $U$ test for continuous variables. We then performed multivariate analysis, with age and gender adjustments, to determine factors independently associated with mortality.

In order to build a predictive model, we used variables at the time of diagnosis of severe dengue as base for variable selection in model-building. Variable selection was performed using five-fold cross-validated Lasso regression. Selected variables were then used to build logistic regression models with cross-validation. Crossvalidation and Lasso addressed overfitting that is known to occur in logistic regression. Logistic regression models built were composed of a combination of 2 of the Lasso-selected variables; and were adjusted for age and gender. All possible combinations were built. Finally, their AUROC were computed to allow comparison between models.

All tests of significance were 2-sided, and we took $p$-value $<0.05$ indicating statistical significance.

\section{Results}

\section{Patient characteristics}

A total of 199 adult patients diagnosed as severe dengue were admitted to the Department of Medicine, Kuala Lumpur Hospital between 1 January 2014 and 31 December 2015 and all were included. All cases had confirmation of dengue infection by NS1 antigen, high-titre IgG or IgM or combination. Of this, 20 patients died.

The clinical characteristics and laboratory parameters are shown in Table 1. Patients who died were significantly older than survivors $(p=0.0003)$. However, only $3 \%$ of total were aged $\geq 60$ years old. Two-thirds of our severe dengue patients were males. More than a third $(34.2 \%)$ was obese $\left(\mathrm{BMI} \geq 27.5 \mathrm{~kg} / \mathrm{m}^{2}\right)$. Co-morbidity was present in almost a third but there was no statistically significant difference between groups. The commonest co-morbidity was diabetes mellitus (12.1\%). Patients who died had a statistically significant larger proportion of patients with multiple co-morbidities compared to survivors $(p=0.04)$. NS1 antigen was positive in two-thirds of patients, high-titre IgG in almost a quarter and more than half were IgM positive. There was no statistically significant difference between groups.

\section{Proportions of abnormal parameters at diagnosis of severe dengue}

Systolic blood pressure $<90 \mathrm{mmHg}$ was present in $22.2 \%$ of patients and there were $16.2 \%$ of patients with SBP
$<90 \mathrm{mmHg}$ who were still febrile (temperature $>38^{\circ} \mathrm{C}$ ). Mean arterial pressure $<65 \mathrm{mmHg}$ was present in $22.7 \%$, diastolic blood pressure $<50 \mathrm{mmHg}$ in $18.7 \%$ and tachycardia defined by pulse rate $>100$ beats $/ \mathrm{min}$ in $47.7 \%$. The proportion of patients diagnosed with shock who had temperature $>38{ }^{\circ} \mathrm{C}$ were $38.9 \%$. Respiratory rate $\geq 25$ breaths per minute occurred in $18.1 \%$ of patients.

Leucopenia was present only in $46 \%$ of patients and platelet count $<20 \times 10^{3} / \mu \mathrm{L}$ in $27.9 \%$. Aspartate transaminase $\geq 1000 \mathrm{U} / \mathrm{L}$ occurred in $21.7 \%$ of patients and alanine transaminase $\geq 1000 \mathrm{U} / \mathrm{L}$ in merely $4.2 \%$. Serum bicarbonate was abnormal $(<21 \mathrm{mmol} / \mathrm{L})$ in $48.4 \%$ of patients whilst serum lactate $>2.0 \mathrm{mmol} / \mathrm{L}$ was detected in $36.9 \%$.

Haematocrit was elevated in $41.3 \%$ of males (Hct > $46 \%$ ) and $43.1 \%$ of females (Hct $>40 \%$ ). Serum creatinine was elevated in $27.2 \%$ of males (serum creatinine > $106 \mu \mathrm{mol} / \mathrm{L}$ ) and in $11.4 \%$ of females (serum creatinine $>96 \mu \mathrm{mol} / \mathrm{L})$.

\section{Temporal relationships}

Timing of clinical events and phases of clinical course are shown in Table 2. Median admission to outcome duration was 8.75 days. Timings of admission, diagnosis of severe dengue, defervescence and outcome occurred earlier in patients who died compared to survivors; however these were not statistically significant. Our study revealed that majority of patients were still febrile (temperature were $>$ $38^{\circ} \mathrm{C}$ ) when they were admitted and when they were diagnosed as severe dengue. These findings were statistically significantly different between groups. Over a third of patients presented as severe dengue upon presentation but no difference were seen between groups.

We also found that nadir platelet occurred almost a day later after nadir WBC in both groups. However, these timings were not statistically significantly different between groups. Similarly, lowest serum bicarbonate occurred before serum lactate peaked however no statistical difference between groups in terms of timings were found.

\section{Factors associated with death: Univariate analysis}

Univariate analyses found 22 clinical and laboratory parameters which were statistically significantly different between patients who died compared to those who survived (Tables 1 and 2). Factors at diagnosis of severe dengue which were associated with mortality were: older age, presence of multiple co-morbidities, presence of lethargy, bleeding, tachycardia, lower platelet count, elevated serum creatinine, elevated AST, elevated ALT, low serum bicarbonate and elevated serum lactate.

Our univariate analyses also showed that mortality was also associated with lower nadir platelet level. In the group that died, highest median serum creatinine was 3 times 
Table 1 Clinical characteristics and laboratory parameters of 199 patients hospitalised with severe dengue in 2014

\begin{tabular}{|c|c|c|c|c|c|c|c|}
\hline & \multicolumn{2}{|c|}{ All $(N=199)$} & \multicolumn{2}{|c|}{ Died $(N=20)$} & \multicolumn{2}{|c|}{ Survived $(N=179)$} & \multirow[t]{2}{*}{$p$-value } \\
\hline & $n$ & $\mathrm{n}(\%)$ or median $(\mathrm{IQR})$ & $n$ & $\mathrm{n}(\%)$ or median(IQR) & $n$ & $\mathrm{n}(\%)$ or median(IQR) & \\
\hline Age, years & 197 & $30.8(24.7-41.3)$ & 20 & $43.5(30.3-54.8)$ & 177 & $30.2(23.9-38.9)$ & 0.0003 \\
\hline BMl & 117 & $24.9(21.3-29.7)$ & 6 & $23.6(22.7-24.1)$ & 111 & $25.3(21.3-30.0)$ & 0.29 \\
\hline Gender, Male & 199 & $127(63.8 \%)$ & 20 & $13(65 \%)$ & 179 & $114(63.7 \%)$ & 0.91 \\
\hline Presence of any co-morbidity & 198 & $62(31.2 \%)$ & 20 & $9(45 \%)$ & 178 & $53(29.6 \%)$ & 0.16 \\
\hline \multicolumn{8}{|l|}{ Co-morbidity } \\
\hline COPD/BA & & $9(4.6 \%)$ & & $0(0 \%)$ & & $9(5.1 \%)$ & \\
\hline DM & & $24(12.1 \%)$ & & $5(25 \%)$ & & $19(10.7 \%)$ & \\
\hline Hypertension & & $9(4.6 \%)$ & & $2(10 \%)$ & & 7 (3.9\%) & \\
\hline $\mathrm{IHD}$ & & $2(1 \%)$ & & $1(5 \%)$ & & $1(0.6 \%)$ & \\
\hline Pregnancy & & $8(4 \%)$ & & $1(5 \%)$ & & 7 (3.9\%) & \\
\hline Others & & $10(5.1 \%)$ & & $0(0 \%)$ & & $10(5.6 \%)$ & \\
\hline Multiple co-morbidities & 198 & $20(10.1 \%)$ & 20 & $5(25 \%)$ & 178 & $15(8.4 \%)$ & $0.04^{\mathrm{a}}$ \\
\hline NS1 & 198 & $132(66.7 \%)$ & 20 & $13(65 \%)$ & 178 & 119 (66.9\%) & 0.87 \\
\hline High-titre lgG & 198 & $47(23.7 \%)$ & 20 & $5(25 \%)$ & 178 & $42(23.6 \%)$ & 0.89 \\
\hline $\lg M$ & 198 & 115 (58.1\%) & 20 & $14(70 \%)$ & 178 & 101 (56.7\%) & 0.25 \\
\hline Persistent vomiting & 198 & 114 (57.6\%) & 20 & $14(70 \%)$ & 178 & $100(56.2 \%)$ & 0.24 \\
\hline Abdominal pain & 198 & $89(45 \%)$ & 20 & $7(35 \%)$ & 178 & $82(46.1 \%)$ & 0.35 \\
\hline Lethargy & 198 & $96(48.5 \%)$ & 20 & $15(75 \%)$ & 178 & $81(45.5 \%)$ & 0.01 \\
\hline Palpable liver & 198 & $16(8.1 \%)$ & 20 & $2(10 \%)$ & 178 & 14 (7.9\%) & $0.67^{\mathrm{a}}$ \\
\hline Fluid accumulation & 198 & $44(22.2 \%)$ & 20 & $4(20 \%)$ & 178 & $40(22.5 \%)$ & $1.00^{\mathrm{a}}$ \\
\hline Bleed & 198 & $49(24.8 \%)$ & 20 & $13(65 \%)$ & 178 & $36(20.2 \%)$ & $<0.0001^{\circ}$ \\
\hline $\mathrm{Hct}>46$ & 198 & 77 (38.9\%) & 20 & $10(50 \%)$ & 178 & $67(37.6 \%)$ & 0.28 \\
\hline Low Plt $<46$ & 198 & 106 (53.5\%) & 20 & $15(75 \%)$ & 178 & $91(51.1 \%)$ & 0.04 \\
\hline Albumin $<34$ & 198 & 106 (53.5\%) & 20 & $13(65 \%)$ & 178 & $93(52.3 \%)$ & 0.28 \\
\hline Number of warning signs & 199 & & 20 & & 179 & & $N S^{b}$ \\
\hline 0 & & $9(4.5 \%)$ & & $0(0 \%)$ & & $9(5.03 \%)$ & \\
\hline 1 & & $17(8.5 \%)$ & & $0(0 \%)$ & & $17(9.5 \%)$ & \\
\hline 2 & & $35(17.6 \%)$ & & $2(10 \%)$ & & $33(18.4 \%)$ & \\
\hline 3 & & $35(17.6 \%)$ & & $4(20 \%)$ & & $31(17.3 \%)$ & \\
\hline 4 & & $49(24.6 \%)$ & & $6(30 \%)$ & & $43(24.0 \%)$ & \\
\hline 5 & & $29(14.6 \%)$ & & $2(10 \%)$ & & $27(15.1 \%)$ & \\
\hline 6 & & $16(8 \%)$ & & $3(15 \%)$ & & $13(7.3 \%)$ & \\
\hline 7 & & $5(2.5 \%)$ & & $0(0 \%)$ & & $5(2.8 \%)$ & \\
\hline 8 & & $3(1.5 \%)$ & & $2(10 \%)$ & & $1(0.6 \%)$ & \\
\hline 9 & & $1(0.5 \%)$ & & $1(5 \%)$ & & $0(0 \%)$ & \\
\hline Presence of any warning signs & 198 & $190(95.5 \%)$ & 20 & $20(100 \%)$ & 178 & 170 (95.0\%) & $1.00^{\mathrm{a}}$ \\
\hline \multicolumn{8}{|l|}{ Severe dengue by type } \\
\hline Decompensated shock & & $58(29.1 \%)$ & & $10(50 \%)$ & & $48(26.8 \%)$ & \\
\hline Compensated shock & & $69(34.7 \%)$ & & $4(20 \%)$ & & $65(36.3 \%)$ & \\
\hline Respiratory compromise & & $42(21.1 \%)$ & & $2(10 \%)$ & & $40(22.3 \%)$ & \\
\hline Severe bleeding & & $24(12.1 \%)$ & & $10(50 \%)$ & & $14(7.8 \%)$ & \\
\hline Severe hepatitis & & $40(20.1 \%)$ & & $8(40 \%)$ & & $32(17.9 \%)$ & \\
\hline AKI & & 35 (17.6\%) & & $8(40 \%)$ & & $27(15.1 \%)$ & \\
\hline
\end{tabular}


Table 1 Clinical characteristics and laboratory parameters of 199 patients hospitalised with severe dengue in 2014 (Continued)

\begin{tabular}{|c|c|c|c|c|c|c|c|}
\hline & \multicolumn{2}{|c|}{ All $(N=199)$} & \multicolumn{2}{|c|}{ Died $(N=20)$} & \multicolumn{2}{|c|}{ Survived $(N=179)$} & \multirow[t]{2}{*}{$p$-value } \\
\hline & $n$ & $\mathrm{n}(\%)$ or median $(\mathrm{IQR})$ & $n$ & $\mathrm{n}(\%)$ or median(IQR) & $n$ & $\mathrm{n}(\%)$ or median(IQR) & \\
\hline Encephalitis & & $6(3.0 \%)$ & & $2(10 \%)$ & & $4(2.2 \%)$ & \\
\hline Other & & $5(2.5 \%)$ & & $1(5 \%)$ & & $4(2.2 \%)$ & \\
\hline Number of types of severe dengue & & & & & & & $N S^{b}$ \\
\hline 1 & & $139(69.8 \%)$ & & $12(60 \%)$ & & $127(70.9 \%)$ & \\
\hline 2 & & $53(26.6 \%)$ & & $4(20 \%)$ & & $49(27.4 \%)$ & \\
\hline 3 & & $5(2.5 \%)$ & & $3(15 \%)$ & & $2(1.1 \%)$ & \\
\hline 4 & & $2(1.0 \%)$ & & $1(5 \%)$ & & $1(0.6 \%)$ & \\
\hline Blood products given & 198 & $57(28.8 \%)$ & 20 & $18(90 \%)$ & 178 & $39(21.9 \%)$ & $<0.0001$ \\
\hline $\mathrm{SBP}, \mathrm{mmHg}$ & 198 & $103(90-120)$ & 20 & $98(87-133)$ & 178 & $103(90-118)$ & 0.88 \\
\hline $\mathrm{DBP}, \mathrm{mmHg}$ & 198 & $63(53-74)$ & 20 & $60.5(48-84)$ & 178 & $63(54-74)$ & 0.92 \\
\hline MAP, $\mathrm{mmHg}$ & 198 & $77(65-89)$ & 20 & $74.3(61-102)$ & 178 & $77.3(66-88)$ & 0.96 \\
\hline PR, beats per minute & 197 & $98(86-110)$ & 20 & $110(102-120)$ & 177 & $96(86-109)$ & 0.01 \\
\hline Shock Index, bpm/mmHg & 197 & $1.2(1.0-1.5)$ & 20 & $1.3(1.0-1.9)$ & 177 & $1.2(1.0-1.4)$ & 0.25 \\
\hline $\mathrm{RR}$, breaths/min & 182 & $20(18-24)$ & 20 & $22(19-30)$ & 162 & $20(18-23)$ & 0.07 \\
\hline $\mathrm{WBC}, \times 10^{3} / \mu \mathrm{L}$ & 198 & $4.2(2.9-5.7)$ & 20 & $5.3(2.7-8.4)$ & 178 & $4.1(2.9-5.6)$ & 0.15 \\
\hline $\mathrm{Hb}, \mathrm{g} / \mathrm{dL}$ & 198 & $14.2(12.3-16.2)$ & 20 & $13.9(11.7-17.1)$ & 178 & $14.2(12.3-16.1)$ & 0.97 \\
\hline $\mathrm{Hct}, \%$ & 198 & $42(37-47.0)$ & 20 & $42.5(31.0-49.5)$ & 178 & $42(37.0-47.0)$ & 0.91 \\
\hline Platelet, $\times 10^{3} / \mu \mathrm{L}$ & 197 & $43(16-97)$ & 20 & $26(8-41)$ & 177 & $53(18-105)$ & 0.005 \\
\hline Serum creatinine, $\mu \mathrm{mol} / \mathrm{L}$ & 195 & $79(63-101)$ & 20 & $110(67-333)$ & 175 & 75 (63-97) & 0.01 \\
\hline AST, U/L & 184 & $165(66-600)$ & 19 & 1175 (123-1920) & 165 & $152(63-456)$ & 0.01 \\
\hline $\mathrm{ALT}, \mathrm{U} / \mathrm{L}$ & 192 & $104(34-257)$ & 20 & $365(62-755)$ & 172 & $95(31-211)$ & 0.01 \\
\hline Troponin T, ng/mL & 17 & $0.02(0.009-0.3)$ & 6 & $0.4(0.05-1.9)$ & 11 & $0.009(0.007-0.03)$ & 0.10 \\
\hline Serum bicarbonate, $\mathrm{mmol} / \mathrm{L}$ & 192 & $21.1(18.5-23.1)$ & 20 & $16.2(11.2-18.8)$ & 172 & $21.5(19.4-23.2)$ & $<0.0001$ \\
\hline Serum lactate, $\mathrm{mmol} / \mathrm{L}$ & 149 & $1.6(1.1-2.3)$ & 19 & $3.2(2.1-6.5)$ & 130 & $1.5(1.1-2.1)$ & $<0.0001$ \\
\hline Nadir WBC, $\times 10^{3} / \mu \mathrm{L}$ & 198 & $2.7(1.94-3.8)$ & 20 & $2.6(2.1-4.1)$ & 178 & $2.7(1.9-3.7)$ & 0.47 \\
\hline Highest Hct, \% & 198 & $45.1(40.9-49.0)$ & 20 & $46.9(41.0-50.2)$ & 178 & $45.0(40.9-49.0)$ & 0.38 \\
\hline Nadir Platelet, $\times 10^{3} / \mu \mathrm{L}$ & 198 & $18(7-44)$ & 20 & $7(2-21)$ & 178 & $19(8-45)$ & 0.005 \\
\hline Highest serum creatinine, $\mu \mathrm{mol} / \mathrm{L}$ & 198 & $87(69-112)$ & 20 & $249(175-378)$ & 178 & $83(68-102)$ & $<0.0001$ \\
\hline Highest AST, U/L & 193 & $262(100-1024)$ & 20 & $4763(658-21,547)$ & 173 & $244(98-589)$ & $<0.0001$ \\
\hline Highest ALT, U/L & 197 & $147(58-450)$ & 20 & $1243(243-3780)$ & 177 & $117(55-303)$ & $<0.0001$ \\
\hline Highest Troponin T, ng/mL & 22 & $0.01(0.005-0.3)$ & 5 & $0.7(0.1-1.9)$ & 17 & $0.01(0.005-0.03)$ & 0.07 \\
\hline Lowest Serum bicarbonate, $\mathrm{mmol} / \mathrm{L}$ & 195 & $19.5(16.9-21.4)$ & 20 & $8.5(6.7-13.1)$ & 175 & $19.9(17.9-21.5)$ & $<0.0001$ \\
\hline Highest Serum lactate, $\mathrm{mmol} / \mathrm{L}$ & 189 & $1.9(1.3-2.8)$ & 20 & $16.0(6.7-18.5)$ & 169 & $1.8(1.2-2.3)$ & $<0.0001$ \\
\hline Total fluids given, $\mathrm{mL}$ & 193 & 6034 (3470-8707) & 20 & $9808(7184-16,692)$ & 173 & $5610(3307-8259)$ & 0.0004 \\
\hline
\end{tabular}

$n$ count of non-missing observations; ${ }^{~}$ Fisher's exact test; ${ }^{b}$ Post-hoc multiple comparisons tests using Scheffe's method; $I Q R$ interquartile range, $B M I$ body mass index, COPD chronic obstructive airway disease, BA bronchial asthma, DM diabetes mellitus, IHD ischemic heart disease, NS1 non-structural protein 1 antigen, $S B P$ systolic blood pressure, $D B P$ diastolic blood pressure, MAP mean arterial pressure, $P R$ pulse rate, $R R$ respiratory rate, $A S T$ aspartate transaminase, $A L T$ alanine transaminase, WBC white blood cell count, Hct hematocrit, AKI acute kidney injury, NS not significant

higher than survivors, highest median AST was almost 20 times higher, highest median ALT was more than 10 times higher, highest median lactate was more than 8 times higher and lowest median serum bicarbonate was more than 2-fold lower. These had statistically significant difference between groups. Non-survivors were found to have received statistically significantly higher volume of fluid
(1.7 times more) and a larger proportion (4.1 times) who received blood products transfusion.

\section{Factors associated with death: Multivariate analysis and predictive model}

Multivariate analysis, with adjustment for age and gender, revealed lethargy, bleeding, pulse rate, serum bicarbonate 
Table 2 Timing of clinical events in 199 patients hospitalised with severe dengue in 2014

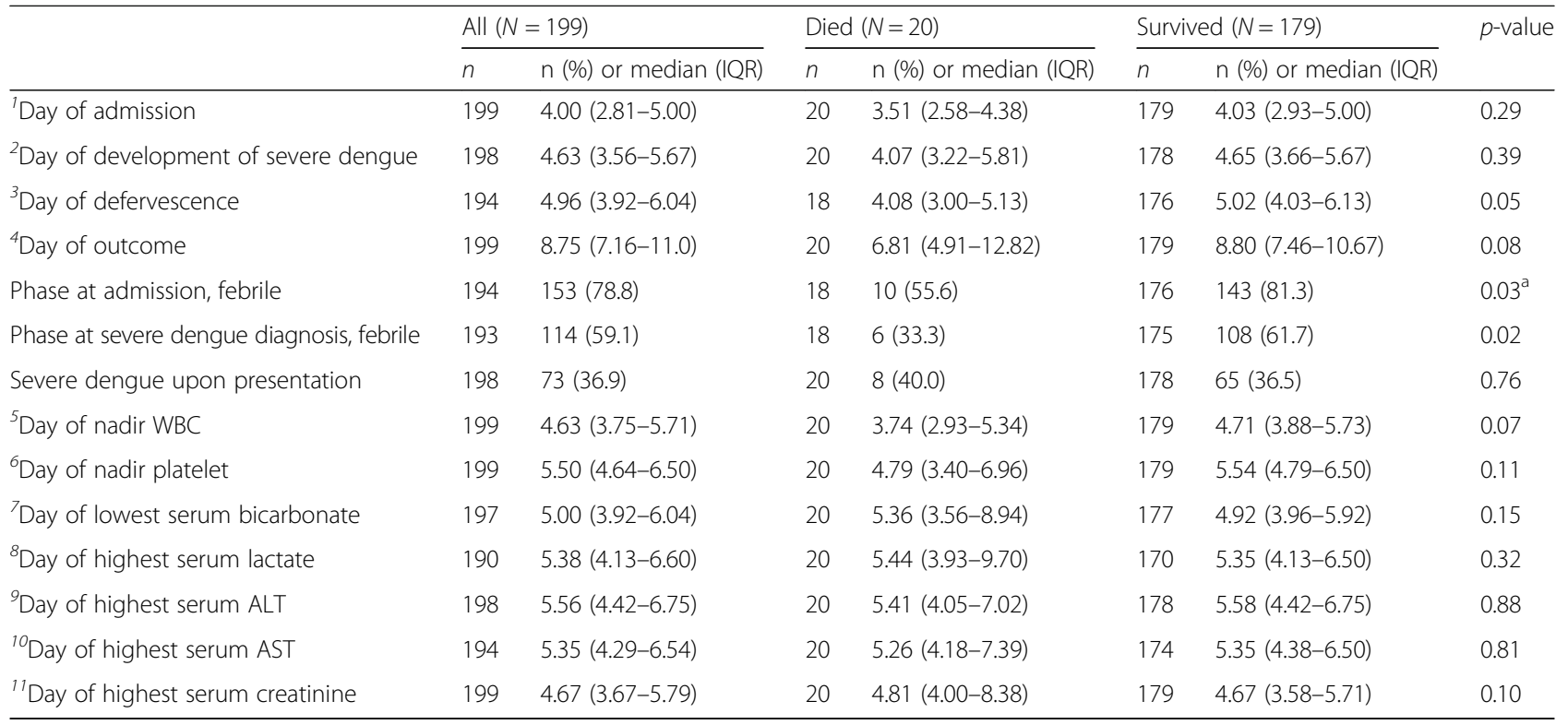

$n$ count of non-missing observations; ${ }^{\text {a}}$ Fisher's exact test; IQR interquartile range, WBC white blood cell count, $A S T$ aspartate transaminase, $A L T$ alanine transaminase;

1-11 These are days from onset of fever

and serum lactate, to be statistically significant as independent predictors of death among severe dengue cases (Table 3).

Lasso selected age, pulse rate, bleeding, serum bicarbonate, serum lactate, serum creatinine, AST and ALT out of 29 candidate variables. Pulse rate, bleeding, serum bicarbonate, serum lactate, serum creatinine, AST and ALT were then used to build models composed of a combination of a pair of these variables, with age and gender adjustments. Twenty-one models were built (Additional file 1: Table S1). We found that serum bicarbonate-based models outperformed lactate-based models (Table 4). The best AUROC was obtained with serum bicarbonate - ALT model. The cross-validated

Table 3 Multivariate analysis of potential factors that predict mortality at the time of diagnosis of severe dengue

\begin{tabular}{llll}
\hline Parameters & Adjusted OR & $95 \% \mathrm{Cl}$ & $p$ \\
\hline Lethargy & 3.84 & $(1.23-12.03)$ & 0.0208 \\
Bleed & 8.88 & $(2.91-27.15)$ & 0.0001 \\
Multiple co-morbidities & 1.28 & $(0.32-5.15)$ & 0.7258 \\
Pulse rate & 1.04 & $(1.01-1.07)$ & 0.0058 \\
Serum bicarbonate & 0.79 & $(0.70-0.89)$ & $<0.0001$ \\
Serum lactate & 1.27 & $(1.09-1.47)$ & 0.0019 \\
PIt & 0.981 & $(0.97-1.00)$ & 0.0231 \\
ALT & 1.001 & $(1.00-1.002)$ & 0.0046 \\
AST & 1.001 & $(1.00-1.001)$ & 0.0152 \\
Serum creatinine & 1.008 & $(1.00-1.01)$ & 0.0044 \\
\hline
\end{tabular}

OR odds ratio, $\mathrm{Cl}$ confidence interval serum bicarbonate - ALT model was found to be significantly different from the uncrossed model (Fig. 1).

\section{Discussion}

Our study made two notable findings: the first being description of aspects of severity of a severe dengue cohort; and the second, the rigorous building of a predictive model, which was selected by assessment and comparison of many models, that predicts death early at the recognition of severe dengue.

Our cohort bears some characteristics of Malaysia's population. The National Health Morbidity Survey 2015 determined that $30.6 \%$ of the population were obese and $17.5 \%$ were diabetics, figures similar to this study [8].

The fatality rate of this study was $10.1 \%$. Previous studies similar to ours had rates of 3.8\% [9] and 18.6\% [10] in Brazil. Amâncio et al. [10] non-survivors had median (IQR) AST 151 (43-474) U/L and mean (SD)

Table 4 Area under curve of receiver operating curve (AUROC) of models for predicting mortality among cases of severe dengue

\begin{tabular}{ll}
\hline Model & AUROC (95\% Cl) \\
\hline Seum bicabote
\end{tabular}

Serum bicarbonate - ALT 83.5 (72.4-94.6)

Serum bicarbonate - Pulse rate

$83.4(73.5-93.4)$

Serum bicarbonate - Serum creatinine

$83.1(72.0-94.3)$

Serum bicarbonate - Bleed

$82.9(70.5-95.2)$

Serum lactate - ALT

$80.8(67.2-94.4)$

Serum lactate - Pulse rate

$78.7(65.7-91.8)$

Serum lactate - Serum creatinine

$77.7(63.9-91.5)$

Serum lactate - Bleed

$81.1(68.2-94.0)$

$A U R O C$ area under curve; $\mathrm{Cl}$ confidence interval 


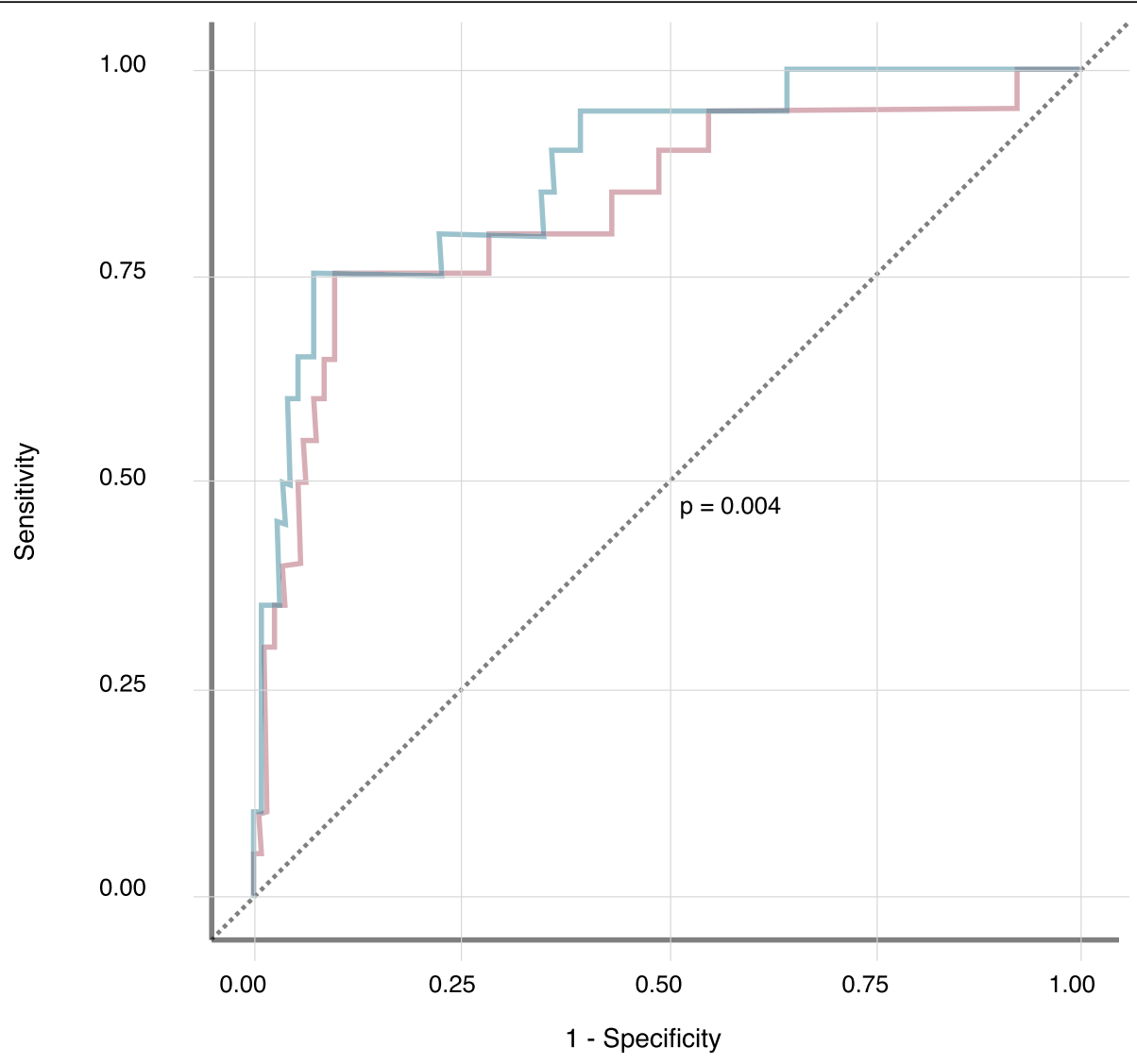

Fig. 1 Cross-validated and uncross-validated (basic) ROC curves of the serum bicarbonate - ALT model

platelet count $83.3 \times 10^{3} / \mu \mathrm{L}\left(76.2 \times 10^{3} / \mu \mathrm{L}\right)$; the other study [9] however, had no similar parameters for comparison. These levels were worse in our study. Other studies, which used ICU admission as the defining criterion of their cohorts, had case fatality rates of $23.1 \%$ [11] in Taiwan whilst in India rates were 11.1\% [12] and 6.1\% [13]. Chen et al. [11] had non-survivors with mean (SD) AST 3444.4 (4191.9) U/L and mean (SD) nadir platelet $3.5 \times 10^{3} / \mu \mathrm{L}\left(4.3 \times 10^{3} / \mu \mathrm{L}\right)$. In contrast, the nonsurvivors of our study had even higher peak AST level, suggesting a more severe cohort in ours.

Our study showed that about a third of patients presented as severe dengue upon admission to hospital whilst the remaining developed severe dengue after admission. Duration of fever onset to severe dengue diagnosis were shorter in those who presented as severe dengue as compared to those who developed severe dengue after admission, median(IQR) 4.38 [2] days vs median(IQR) 5.02(1.78) days, $p=0.003$, respectively. Interestingly, there were no difference in mortality and in the duration of fever onset to admission between those who presented as severe dengue as compared to those who developed severe dengue after admission, $p=0.95$ vs $p=0.29$, respectively.
Guidelines in dengue have repeatedly highlighted that the timing of deterioration is around the time of defervescence and within the critical phase that ensues. It has also been noted that organ impairment follows the same timing. Intriguingly, we found that almost $60 \%$ of patients were still febrile at diagnosis of severe dengue; and the proportion of patients who were still febrile at diagnosis of severe dengue was statistically significantly more in those who survived as compared to those who died (Table 2). In fact, those who were febrile at diagnosis of severe dengue were less likely to die as compared to those who had defervesced, OR 0.29 (95\% CI: 0.09-0.92, $p=0.03$ ). The importance of timing of development of severe dengue in the course of illness needs to be further explored.

Our findings that nadir platelet occurred about a day after nadir WBC is consistent with a previous study [14]. However, another intriguing finding of our study is that the timing of peak serum creatinine coincided with nadir WBC in those who survived whereas in those who died it coincided with the later nadir platelet.

Based on our cohort, independent predictors of death at the time when the diagnosis of severe dengue was made were: lethargy, bleeding, pulse rate, serum bicarbonate 
and serum lactate. There have been only 2 studies so far, that examined factors associated with death among severe dengue patients, which used WHO 2009 classification. A study utilising notification database from Brazil [9] of mixed age groups showed age $>55$ years (OR 4.98), gastrointestinal bleeding (OR 10.26), haematuria (OR 5 . 07), and thrombocytopenia (OR 2.55) were factors associated with death. However, exact timings of these parameters were not included. A second study [10] was of severe dengue patients admitted to ICU in Brazil. In this study of 97 patients admitted to ICU, parameters taken within $24 \mathrm{~h}$ of ICU admission that were found to be associated with death were: comorbidity of chronic renal disease (OR 15.6), presence of persistent vomiting (OR 4.25), lethargy (OR 3.23), dyspnoea (OR 3.27), elevated WBC, higher serum creatinine and lower serum albumin.

We found that the best prediction model to predict death at the time when the diagnosis of severe dengue was made is a model that incorporated serum bicarbonate and ALT levels taken at that time. This is rather fortuitous for a few reasons. Firstly, both serum bicarbonate and ALT are objective measures as opposed to subjective warning signs such as lethargy and bleeding which have inherent variability in establishing their presence and severity. Secondly, both are currently accessible laboratory tests.

A recent study on serum lactate in dengue [15] found that this biomarker is a good predictor of severe dengue (AUROC for peripheral venous lactate at admission was 0.84 [95\% CI: 0.72-0.97]). In perspective, our study revealed that in predicting death, lactate-incorporated models had lower AUROCs as compared to those of bicarbonate-based models (Table 4). Additionally, we showed that nadir serum bicarbonate occurred earlier than highest serum lactate level (Table 2) in patients with severe dengue. In fact, at diagnosis of severe dengue, more patients had abnormal serum bicarbonate than abnormal serum lactate $(46.7 \%$ vs $27.6 \%$, respectively). These are important aspects to consider in clinical practice as earlier management will be more advantageous. Therefore, whilst lactate could be an additional alternative criterion to establish the diagnosis of severe dengue, our study suggests that lactate alone is not sufficient in prognostication of patients with severe dengue.

Unexpectedly, serum creatinine was not found to be an independent predictor of death by multivariate analysis. However, its incorporation into our models led to good AUROC performances.

Under the assumption that the time of recognition of severe dengue is approximately the actual time of development of severe dengue, we believe that prognosticating mortality in patients with severe dengue at the time of its recognition provides a sensible approach. We postulate that at this time, underlying pathophysiological processes which determine outcome would most likely have reach significance. Prognosticating prematurely before this moment may have little specificity, as the ultimate outcome determining processes may yet to occur. Prognosticating too late however is obviously futile. With our model management decisions may be better informed in terms of resource allocation, especially in conditions of high volume care. It has to be noted however that the underlying outcome determining pathophysiology has yet to be clearly elucidated. Further investigation into the kinetics of biochemistry with respect to timing of events in dengue, in particular the time of development of severe dengue, is needed and may perhaps fill this gap.

The main limitation of our study was the retrospective design. However, data accuracy was reasonable as management of patients followed standard management guidelines for dengue which have clear specifications of timing of blood investigations. Though only a single centre study, we believe the sample size was adequate as illustrated by the results of our findings. Finally, though we have rigorously built a predictive model and took steps to address overfitting, the actual performance of any model will vary according to the population it is applied on. As we have mentioned, our cohort is different from similar studies, hence our model will require population specific external validation and assessment.

\section{Conclusions}

In conclusion, the degree of severity observed in this study was more serious than those reported in similar studies. We showed that a large proportion of severe dengue occurred early, whilst patients were still febrile. Finally, the regression equation to our model is: Log odds of death among severe dengue cases $=-1$. $021-0.220$ (Serum bicarbonate) $+0.001($ ALT $)+0.067$ (Age) - 0.190(Gender).

\section{Additional file}

Additional file 1: Table S1. AUROC of all logistic regression model combinations. The table contains areas under curve of receiver operating curves (AUROC) of all logistic regression models built using pairwise combinations of LASSO-selected variables. All possible pairwise combinations were made. Each model was adjusted for age and gender. Models were listed from highest to lowest AUROC. (DOCX $9 \mathrm{~kb}$ )

\section{Abbreviations}

ALT: Alanine transaminase; AST: Aspartate transaminases; AUROC: Area under curve of receiver operating curve; Cl: Confidence interval; DENV: Dengue virus; ICU: Intensive care unit; IgG: Immunoglobulin G; IgM: Immunoglobulin M; IQR: Interquartile range; MAP: Mean arterial blood pressure; MREC: Medical Research and Ethics Committee; NMRR: National Medical Research Register; NS1: Non-structural protein 1; OR: Odds ratio; SBP: Systolic blood pressure; SD: Standard deviation; STROBE: STrengthening the Reporting of OBservational studies in Epidemiology; WBC: White blood count; WHO: World Health Organization 


\section{Acknowledgements}

The authors would like to thank all the staff and nurses of Dengue Wards, Department of Medicine, Kuala Lumpur Hospital and the Records Office, Kuala Lumpur Hospital for their assistance in the handling of medical records. We also would like to thank the Director General of Health Malaysia for his permission to publish this article.

\section{Availability of data and materials}

Data are available upon direct request to the corresponding author, Dr. Julina MD-NOOR, julinamn@gmail.com, subject to approval by the Director-General of Health, Ministry of Health, Malaysia.

\section{Authors' contributions}

Concept and design: Md-Sani, Md-Noor, Han, Abd-Rahman. Acquisition, analysis, or interpretation of data: Md-Sani, Han, Gan, Rani, Tan, Rathakrishnan, A-Shariffuddin. Drafting of the manuscript: Md-Sani, Md-Noor, Han. Critical revision of the manuscript for important intellectual content: Md-Sani, Md-Noor, Abd-Rahman, Han. Administrative, technical, or material support: Md-Sani, Md-Noor, A-Shariffuddin. All authors read and approved the final manuscript.

\section{Ethics approval and consent to participate}

The study was approved by the Medical Research and Ethics Committee (MREC), Ministry of Health of Malaysia (Research ID NMRR-15-2023-24,849). As the study involved data collection from case notes only, the MREC granted a waiver of informed consent.

\section{Consent for publication}

Not applicable as the study involved data collection from case notes only, the MREC (Medical Research and Ethics Committee) granted a waiver of informed consent (Research ID NMRR-15-2023-24,849).

\section{Competing interests}

The authors declare that they have no competing interests.

\section{Publisher's Note}

Springer Nature remains neutral with regard to jurisdictional claims in published maps and institutional affiliations.

\section{Author details}

'Department of Medicine, Hospital Kuala Lumpur, Jalan Pahang, 50586 Kuala Lumpur, Malaysia. ${ }^{2}$ Faculty of Medicine, Universiti Teknologi MARA (UiTM), Jalan Hospital, 47000 Sungai Buloh, Selangor, Malaysia. ${ }^{3}$ Clinical Research Centre, Hospital Kuala Lumpur, Jalan Pahang, 50586 Kuala Lumpur, Malaysia.

Received: 30 December 2017 Accepted: 10 May 2018

Published online: 21 May 2018

\section{References}

1. Bhatt S, Gething PW, Brady OJ, Messina JP, Farlow AW, Moyes CL, Myers MF. The global distribution and burden of dengue. Nature. 2013:496(7446):504-7.

2. Yusoff, H. M. (2008). National dengue programme in Malaysia. In Asia-Pacific dengue programme managers meeting, 3-9 May 2008, Singapore (pp. 83-86).

3. Mohd-Zaki AH, Brett J, Ismail E, L'Azou M. Epidemiology of dengue disease in Malaysia (2000-2012): a systematic literature review. PLoS Negl Trop Dis. 2014;8(11):e3159.

4. Thayan, R. Dengue - Understanding The Virus. Retrieved from http://jknj. moh.gov.my/jsm/day3/. Accessed 142018

5. Malaysia - Dengue Incidence Rate \& Case Fatality Rate for Year 2000-2015 Retrieved from http://idengue.remotesensing.gov.my/idengue/content/ statistik.pdf. Accessed 142018.

6. Alexander N, Balmaseda A, Coelho IC, Dimaano E, Hien TT, Hung NT, Siqueira JB. Multicentre prospective study on dengue classification in four south-east Asian and three Latin. Trop Med Int Health. 2011;16(8):936-48.

7. John DV, Lin YS, Perng GC. Biomarkers of severe dengue disease-a review. J Biomed Sci. 2015;22(1):83

8. Malaysia, National Institutes of Health, Ministry of Health Malaysia, Institute for Public Health. (2015). National Health and morbidity survey 2015 (NHMS 2015). Vol. II: Non-Communicable Diseases, Risk Factors \& Other Health Problems. Kuala Lumpur: Institute for Public Health.
9. Pinto RC, DBd C, BCd A, Sampaio VS, RAd P, CFd C, et al. Mortality predictors in patients with severe dengue in the state of Amazonas, Brazil. PLoS One. 2016: 11(8):e0161884.

10. Amâncio FF, Heringer TP, CdCHBd O, Fassy LB, FBd C, Oliveira DP, et al. Clinical profiles and factors associated with death in adults with dengue admitted to intensive care units, Minas Gerais, Brazil. PLoS One. 2015;10(6): e0129046.

11. Chen CM, Chan KS, Yu WL, Cheng KC, Chao HC, Yeh CY, Lai CC. The outcomes of patients with severe dengue admitted to intensive care units. Medicine. 2016;95(31):e4376.

12. Chandralekha GP, Trikha A. The north Indian dengue outbreak 2006: a retrospective analysis of intensive care unit admissions in a tertiary care hospital. Trans R Soc Trop Med Hyg. 2008;102:143-7.

13. Juneja $D$, Nasa $P$, Singh $O$, et al. Clinical profile, intensive care unit course, and outcome of patients admitted in intensive care unit with dengue. J Crit Care. 2011;26:449-52.

14. Tricou V, Minh NN, Farrar J, Tran HT, Simmons CP. Kinetics of viremia and NS1 Antigenemia are shaped by immune status and virus serotype in adults with dengue. PLoS Negl Trop Dis. 2011;5(9):e1309.

15. Thanachartwet $V$, Wattanathum A, Oer-Areemitr N, Jittmittraphap A Sahassananda D, Monpassorn C, Desakorn V. Diagnostic accuracy of peripheral venous lactate and the 2009 WHO warning signs for identifying severe dengue in Thai adults: a prospective observational study. BMC Infect Dis. 2016;16(1)

\section{Ready to submit your research? Choose BMC and benefit from:}

- fast, convenient online submission

- thorough peer review by experienced researchers in your field

- rapid publication on acceptance

- support for research data, including large and complex data types

- gold Open Access which fosters wider collaboration and increased citations

- maximum visibility for your research: over $100 \mathrm{M}$ website views per year

At BMC, research is always in progress.

Learn more biomedcentral.com/submissions 